
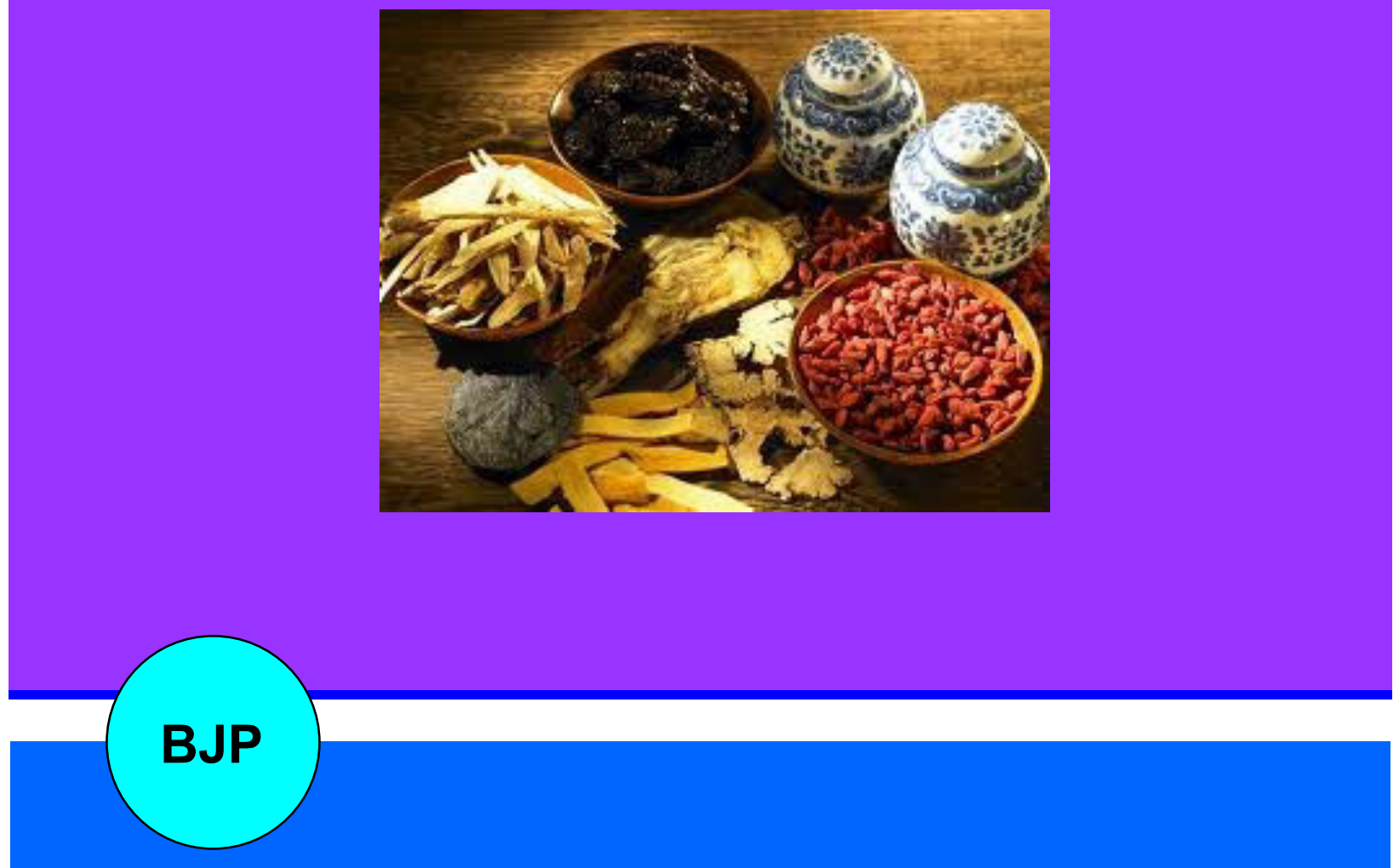

Bangladesh Journal of Pharmacology

Research Article

Pharmacological mechanism of a drug pair of astragali and cinnamon in shiquan dabu decoction 
Abstracted/indexed in Academic Search Complete, Agroforestry Abstracts, Asia Journals Online, Bangladesh Journals Online, Biological Abstracts, BIOSIS Previews, CAB Abstracts, Current Abstracts, Directory of Open Access Journals, EMBASE/Excerpta Medica, Google Scholar, HINARI (WHO), International Pharmaceutical Abstracts, Open J-gate, Science Citation Index Expanded, SCOPUS and Social Sciences Citation Index

\title{
Pharmacological mechanism of a drug pair of astragali and cinnamon in shiquan dabu decoction
}

\author{
Hui Yan, Mingzhu Jiang, Qiongying Wu and Zhongzheng Gui
}

School of Biological and Chemical Engineering, Jiangsu University of Science and Technology, Zhenjiang 212018, Jiangsu, China.

\begin{tabular}{|c|c|}
\hline \multicolumn{2}{|l|}{ Article Info } \\
\hline Received: & 23 May 2011 \\
\hline Accepted: & 4 June 2011 \\
\hline Available Online: & 5 June 2011 \\
\hline \multicolumn{2}{|c|}{ DOI: 10.3329/bjp.v5i2.7769 } \\
\hline \multicolumn{2}{|c|}{$\begin{array}{l}\text { Cite this article: } \\
\text { Yan H, Jiang M, Wu Q, Gui Z. Phar- } \\
\text { macological mechanism of a drug } \\
\text { pair of astragali and cinnamon in shi- } \\
\text { quan dabu decoction. Bangladesh J } \\
\text { Pharmacol. 2010; 5: 107-11. }\end{array}$} \\
\hline
\end{tabular}

\begin{abstract}
The pharmacological mechanism of drug pair of astragali and cinnamon in shiquan dabu decoction were studied in blood deficiency and Qi deficiency modeled mice. In Qi deficiency, the drug pair of astragali and cinnamon in shiquan dabu decoction had the ability of improving the number of white blood cells and peyer patches, and T-cell activity. It had no action in red blood cell number and phagocytic activity of peritoneal macrophage, and Bcell activity. In blood deficiency, the drug pair of astragali and cinnamon in shiquan dabu decoction had the effect of improving the hemoglobin content and T-cell activity. It did not affect the number of white blood cells and peyer patches, phagocytic activity of peritoneal macrophage and B-cell activity. In conclusion, the drug pair of astragali and cinnamon in shiquan dabu decoction has different actions, the quantity of drug pair in shiquan decoction should be changed according to the symptoms in process of diseases.
\end{abstract}

\section{Introduction}

Compound recipe is the mainly used form of Chinese medicine in the prevention and treatment of diseases (Cao et al., 2008; Song et al., 2002; Li et al., 2004). There are two main methods in the study on pharmacological mechanism of compound recipe, which are the whole and separation. The latter including of single herb, drug group and drug pair is beneficial for clarifying the mechanism, and is widely used.

Drug pair consists of two Chinese medicines. Under the guidance of theory of traditional Chinese medicine (TCM) and on the basis of drug compatibility, the two medicines are often used together in prescriptions, e.g., ginseng is usually combined with astragalus, Gypsum and anemarrhena was usually used at the same time (Chen et al., 2007).

Bazhen decoction is used in the treatment of both Qi and blood deficiency (deficiency is a description for symptoms in Chinese medicine). Qi deficiency has symptoms of pale face, dizziness, blurred vision, shortness of breath, less words, mental fatigue, and weakness (Sun, 1993; Liu et al., 2009). Blood deficiency has symptoms of yellowish face, dizziness, limbs malaise, short of breath, few words, palpitation, feeble pulse (Chen et al., 2009; You et al., 2009). More diseases, such as chronic diarrhea, chronic blood loss and so on, can lead to qi deficiency and/or blood deficiency.

Bazhen decoction is made up of 8 Chinese medicines, angelica, ligusticum wallichii, radix paeoniae Alba, rehmannia, ginseng, atractylis ovata, tuckahoe and liquorice. When a drug pair of astragali and cinnamon is added in bazhen decoction, another decoction Shiquan dabu is formed. Shiquan dadu decoction also had good action in the treatment of both qi and blood deficiency (Yang et al., 1986). However, the effect of this drug pair in shiquan dabu decoction is not known at present.

In this work, the pharmacology mechanism of drug pair of astragali and cinnamon in shiquan dabu decoction was explored. 


\section{Materials and Methods}

\section{Instrument}

The UV-VIS spectrophotometer (UV-9100, Beijing Rayleigh Analytical Instruments, China), hemoglobin analyzer (Hb-1002, Shanghai Scientific Instrument Co., Ltd. China) and ELISA Microplate Reader (DG5031, East China Medical Equipment Electronics Group Co., Ltd. China) were used.

\section{Chemical reagents}

RPMI-1640 was provided by Gbico Ltd. (USA), lipopolysaccharide (LPS) were provided by the Sigma Ltd. (USA), cyclophosphamide were purchased from General Pharmaceutical Factory of Harbin Pharmaceutical Group (China), calf serum was purchased from Hangzhou Evergreen Biological Engineering Materials Co., Ltd. (China), hemoglobin determination kit was purchased from Shanghai Rongsheng Biotechnology Ltd. (China). Con A was purchased from Shanghai suolaibao Biological Technology Co. Other chemicals used in this study were of analytical grade.

\section{Chinese medicine}

All of the Chinese medicines were purchased from Guotai great dispensary (Zhenjiang, China).

\section{Animals}

Animals used for study were obtained from Jiangsu University laboratory animal Center. Kunming mice weighing $20 \pm 2 \mathrm{~g}$ each, equal male and female, were housed in polypropylene cages after acclimatization for a period of one week in a new environment. Mice were kept at an ambient temperature and had free access to food and water.

\section{Decoction preparations}

According to the ancient medical book "Zhenti Zhenyao", shiquan dabu decoction consists of $7.8 \mathrm{~g}$ of each astragali and cinnamon, and $11.1 \mathrm{~g}$ each of other 8 Chinese medicines. In this work, Chinese medicines at the same rate aforementioned were put into an enamel pan, and water approximately 5-7 times of the medicine quantity was added. The medicines were soaked in water for 1-2 hours, and were slowly fired for an hour after boiled, then filtered, repeated once, and the filtrate were merged and concentrated into $1 \mathrm{~g}$ (Chinese herbal medicines) per $\mathrm{mL}$ by heating in water bath. In bazhen decoction, weight of 8 kinds of medicine were equal, the preparation method was same as shiquan dabu decoction.

\section{Experimental design}

Mice were randomly divided into 7 groups of each ten, normal group (A), qi deficiency group (B), blood deficiency group (C), qi deficiency group intervened with bazhen decoction (D), qi deficiency intervened with shiquan dabu decoction (E), blood deficiency intervened with bazhen decoction $(F)$ and blood deficiency intervention group with shiquan dabu decoction $(G)$.

\section{Building Qi deficiency model}

Each mouse of group B, D and E was administrated (i.g.) with $1 \mathrm{~g} / \mathrm{mL}$ rhubarb soup at dose of $0.025 \mathrm{~g} / \mathrm{g}$ (w/ $\mathrm{w}$, Chinese medicine/body weight) for 16 days. On the $8^{\text {th }}$ or $9^{\text {th }}$ day, mice began to lose stool, fur turned dim, dispirited and inert, reduced autonomic activities, etc., which suggested that the Qi deficiency model was successfully built. The mouse in group D and E was administrated (i.g.) with shiquan dabu decoction $\left(0.0135 \mathrm{~mL} / \mathrm{g}\right.$ body weight) every afternoon from the $9^{\text {th }}$ day to $16^{\text {th }}$, group B was treated with distilled water.

\section{Building blood deficiency model}

Each mouse of group C, F and G was intraperitoneal injection of cyclophosphamide $0.01 \mathrm{mg} / \mathrm{g}$ body weight on the $1^{\text {st }}, 4^{\text {th }}$ and $7^{\text {th }}$ day to induce the blood deficiency. The mice became sluggish; fur turned dim and began to fall off, which suggested that blood deficiency model were built. Meanwhile, the mice of group F and G had been were administrated (i.g.) with Shiquan dabu decoction $(0.0135 \mathrm{~mL} / \mathrm{g}$ body weight) at afternoon for 8 days.

\section{Blood assay}

Blood was collected from orbital. The number of red blood cells (RBC) and white blood cells (WBC) were counted with a pool of blood counts. Hemoglobin content was measured with hemoglobin meter. Collected blood was in a water bath at $37^{\circ} \mathrm{C}$ for $30 \mathrm{~min}$, centrifuged with $3000 \mathrm{rpm}$ for $5 \mathrm{~min}$, and serum was drawn and stored at $-20^{\circ} \mathrm{C}$ for further test.

\section{Phagocytic activity of peritoneal macrophages}

Mice were intraperitoneal injection of $1 \%(\mathrm{v} / \mathrm{v})$ chicken erythrocyte suspension $1 \mathrm{~mL}$ on next day after the last day of intervened with decoction, 40 min later, mice were died for blood collected, peritoneal cavity were opened and flushed with some physiological saline, then the peritoneal fluid were sucked out and put on a slide, was dried and fixed with methanol for $10 \mathrm{~min}$, then was dyed with gimosa. The total and phagocytic macrophages were counted under microscope. The phagocytic coefficient was calculated by the amount of macrophage that devour chicken WBC divided by total amount of macrophage.

\section{Peyer patche number}

Peyer patche (PP) usually appeared white and located at the intestinal wall opposite to the mesentery. They are unequal in size, and are round or orbicular-ovate. The number of PP, diameter larger than $2 \mathrm{~mm}$, was counted. 


\begin{tabular}{|c|c|c|c|c|c|c|c|}
\hline \multicolumn{8}{|c|}{ Table I } \\
\hline \multicolumn{8}{|c|}{ The influence of the Shiquan dabu decoction to the Qi deficiency model } \\
\hline Group & $\begin{array}{c}\text { RBC } \\
\left(10^{9} / \mathrm{mm}^{3}\right)\end{array}$ & $\begin{array}{c}\text { WBC } \\
\left(10^{9} / \mathrm{mm}^{3}\right)\end{array}$ & $\begin{array}{l}\text { Hemoglobin } \\
(\mathrm{g} / \mathrm{L})\end{array}$ & PP & $\begin{array}{l}\text { Phagocytic } \\
\text { coefficient } \\
(\%)\end{array}$ & $\begin{array}{c}\text { T-cell activity } \\
\text { (A.U) }\end{array}$ & $\begin{array}{c}\text { B-cell } \\
\text { Activity } \\
\text { (A.U.) }\end{array}$ \\
\hline $\mathrm{A}$ & $6.1 \pm 0.5$ & $9.1 \pm 0.3$ & $169.4 \pm 13.4$ & $5.5 \pm 1.2$ & $0.8 \pm 0.1$ & $0.2 \pm 0.0$ & $0.2 \pm 0.0$ \\
\hline B & $4.1 \pm 0.2^{\mathrm{a}}$ & $7.4 \pm 1.3^{a}$ & $132.2 \pm 9.1^{\mathrm{a}}$ & $3.4 \pm 1.2^{\mathrm{a}}$ & $0.5 \pm 0.1^{\mathrm{a}}$ & $0.1 \pm 0.0^{\mathrm{a}}$ & $0.1 \pm 0.0^{\mathrm{a}}$ \\
\hline $\mathrm{D}$ & $5.6 \pm 0.6^{b}$ & $12.4 \pm 1.9^{\mathrm{a}, \mathrm{b}}$ & $151.6 \pm 8.0^{\mathrm{a}, \mathrm{b}}$ & $3.5 \pm 1.0^{\mathrm{a}}$ & $0.7 \pm 0.0^{\mathrm{a}, \mathrm{b}}$ & $0.2 \pm 0.0^{\mathrm{b}}$ & $0.2 \pm 0.0^{b}$ \\
\hline $\mathrm{E}$ & $5.4 \pm 0.2^{\mathrm{a}, \mathrm{b}}$ & $14.0 \pm 1.4^{\mathrm{a}, \mathrm{b}, \mathrm{c}}$ & $140.1 \pm 9.2^{a, b, c}$ & $4.9 \pm 1.5^{\mathrm{b}, \mathrm{c}}$ & $0.8 \pm 0.1^{b}$ & $0.2 \pm 0.0^{\mathrm{a}, \mathrm{b}, \mathrm{d}}$ & $0.2 \pm 0.0^{b}$ \\
\hline
\end{tabular}

\begin{tabular}{|c|c|c|c|c|c|c|c|}
\hline \multicolumn{8}{|c|}{ Table II } \\
\hline \multicolumn{8}{|c|}{ Effect of drug pair on mice with blood deficiency } \\
\hline Group & $\begin{array}{c}\text { RBC } \\
\left(10^{9} / \mathrm{mm}^{3}\right)\end{array}$ & $\begin{array}{c}\text { WBC } \\
\left(10^{3} / \mathrm{mm} 3\right)\end{array}$ & $\begin{array}{l}\mathrm{Hb} \\
(\mathrm{g} / \mathrm{L})\end{array}$ & PP & $\begin{array}{c}\text { Phagocytic } \\
\text { coefficient (\%) }\end{array}$ & $\begin{array}{l}\text { T-cell activity } \\
\text { (A.U.) }\end{array}$ & $\begin{array}{l}\text { B-cell activity } \\
\text { (A.U.) }\end{array}$ \\
\hline $\mathrm{A}$ & $6.1 \pm 0.6$ & $9.1 \pm 0.3$ & $169.4 \pm 13.4$ & $5.5 \pm 1.2$ & $0.8 \pm 0.1$ & $0.2 \pm 0.1$ & $0.2 \pm 0.0$ \\
\hline C & $2.9 \pm 0.2 \mathrm{a}$ & $7.4 \pm 1.2^{\mathrm{a}}$ & $111.2 \pm 10.7^{a}$ & $3.0 \pm 0.8^{a}$ & $0.3 \pm 0.1^{a}$ & $0.1 \pm 0.1^{\mathrm{a}}$ & $0.2 \pm 0.0^{a}$ \\
\hline G & $5.0 \pm 0.3^{\mathrm{a}, \mathrm{c}}$ & $12.1 \pm 1.2^{\mathrm{a}, \mathrm{c}}$ & $129.7 \pm 12.7 \mathrm{a}, \mathrm{c}$ & $6.2 \pm 1.0 c$ & $0.9 \pm 0.1^{c}$ & $0.2 \pm 0.0^{\mathrm{b}}$ & $0.2 \pm 0.0 c$ \\
\hline $\mathrm{F}$ & $5.1 \pm 0.3 \mathrm{a}, \mathrm{c}$ & $10.7 \pm 1.9 \mathrm{c}$ & $154.0 \pm 11.3 \mathrm{a}, \mathrm{c}, \mathrm{d}$ & $6.9 \pm 1.8^{c}$ & $0.9 \pm 0.1 \mathrm{a}, \mathrm{c}$ & $0.2 \pm 0.0^{c, d}$ & $0.2 \pm 0.0 c$ \\
\hline
\end{tabular}

\section{Splenic T, B-cell proliferation}

The spleen was taken out under sterile condition and put into a sterile petri dish, $5 \mathrm{~mL}$ Hanks solution was added, and then the spleen was grinded with tube bottom on a 200 copper mesh. The mixture was collected into the centrifuge tube and centrifuged at $3000 \mathrm{rpm}$ for $10 \mathrm{~min}$, supernatant was removed, a little of Tris- $\mathrm{NH}_{4} \mathrm{Cl}$ was added to swell RBC. Centrifuged and removed the supernatant, added $0.5 \mathrm{~mL}$ PRI-1640 nutrient solution, measured the concentration of cells with trypan blue dye under microscope. The cell concentration was adjusted to $1 \times 10^{6}$ cells $/ \mathrm{mL}$ with nutrient PRI-1640 solution. $200 \mu \mathrm{L}$ splenic cells suspension was added to cell culture plate, then $20 \mu \mathrm{L}$ ConA or $20 \mu \mathrm{L}$ LPS was added. The plates were cultivated in $\mathrm{CO}_{2}$ incubators at $37^{\circ} \mathrm{C}$ with $5 \% \mathrm{CO}_{2}, 44$ hours later, supernatant was disposed, $10 \mu \mathrm{L}$ of $5 \mathrm{mg} /$ mL MTT was added in darkness, and the culture was continued for 4 hours. 100 DMSO was added to each well and fully mixed. 30 min latter, the absorbance value was measured with ELISA Microplate Reader at wavelength of $570 \mathrm{~nm}$.

\section{Statistics analytical}

Data was analyzed with SPSS 13.0 software. The differences among different groups were analyzed using one-way analysis of variance (ANOVA), differences were considered to be statically significant at $\mathrm{p}<0.05$.

\section{Results}

The Chinese medicine theory thinks that repeated diarrhea will cause Qi deficiency. Rhubarb as a kind of traditional Chinese medicine was used to discharge blood stasis, remove lump, clear gastrointestinal tract. Besides, it could improve the gastrointestinal blood perfusion, enhance gastric motility and induce diarrhea. Therefore, repeated administration of rhubarb has become a method used to build qi deficiency model. The result of pharmacologic action of drug pair in shiquan dabu decoction was shown in Table I.

The number of WBC and RBC, hemoglobin content in mice with qi deficiency were significant lower than the normal $(p<0.01)$. After treatment with bazhen or shiquan dabu decoction, they were all increased. For Group D and E, it had significant difference to group B $(p<0.01)$, which suggested that these two decoctions had effect of improving the level of WBC, $\mathrm{RBC}$ and $\mathrm{Hb}$ in the status of Qi deficiency. For WBC and $\mathrm{Hb}$, group $\mathrm{E}$ had significant difference to group D $(\mathrm{p}<0.05)$, which suggested that the drug pair of astragali and cinnamon in shiqu dabu had effect of enhancing WBC and reducing the content of $\mathrm{Hb}$ respectively.

The number of PP in mouse of qi deficiency was significant lower than normal $(\mathrm{p}<0.01$; Table II), and was not significant increase after being treated with bazhen decoction ( $p>0.05)$. However, it increased after being treated with shiquan dabu decoction $(p<0.01)$; 
There were significant difference between bazhen and shiquan decoction $(\mathrm{p}<0.05)$, which suggested that drug pair of astragali and cinnamon had ability of enhancing PP number in shiquan dabu decoction.

Pagocytic coefficient of mice with qi deficiency were lower than normal $(\mathrm{p}<0.01)$, and increased after treatment of the two decoction $(\mathrm{p}<0.01)$. Shiquan dabu improved the pagocytic activity to normal $(p>0.05)$, which suggested that the drug pair had strong effect of improving the phagocytic activity of peritoneal macrophages.

Splenic T, B-cells activity in qi deficiency mice were significant lower than normal mice $(p<0.01)$. For T-cell activity, after treatment of these two decoction, they were all improved and had significant difference to group B $(p<0.01)$. For group $E$, it had significant difference to group A and D, which suggested that the drug pair of astragali and cinnamon in shiqu dabu decoction had vital ability to improve the T-cell activity. For B-cell activity, bazhen and shiquan dabu decotion had ability to improve it to normal level $(p>0.05)$. However, there were no significant difference between group $\mathrm{D}$ and $\mathrm{E}$, which suggested the drug pair of astragali and cinnamon had no effect of improve the Bcell activity in shiquan dabu decoction.

In status of blood deficiency, the number of RBC and WBC and content of $\mathrm{Hb}$ were lower than it in normal mice, and was significant different to normal group $(p<0.01$; Table II). When mice of qi deficiency were intervened by bazhen or shiquan dabu decoction, they all increased $(\mathrm{p}<0.01)$. For $\mathrm{Hb}$, it was enhanced, and had significant difference to group $G(p<0.01)$, it can be inferred that the main action of drug pair astragali and cinnamon in shiquan dabu decoction was the improvement of the $\mathrm{Hb}$ content.

The number of PP in blood deficiency group was lower than normal $(\mathrm{p}<0.01)$, and was increased after it was administration of bazhen or shiquan dabu decoction $(p<0.01)$. Compared with normal group, they were not significant difference $(p>0.05)$. There were no significant difference between group $G$ and $F$, which suggested that the drug pair had no action in improving PP number in shiquan dabu decoction.

Peritoneal macrophage phagocytic coefficient in blood deficiency was lower than normal group $(p<0.01)$, it increased after it was administrated two kinds of decoctions $(p<0.01)$. For group $G$, there was no significant difference to normal group $(p>0.05)$. For group $F$, it was more than normal group and was significant different to it, which suggested that the drug pair of astragali and cinnamon had synergism with bazhen dececotin in enhancing the phagocytic activity of peritoneal macrophage.

In status of blood deficiency, splenic T, B activities were declined, and increased when was administrated with bazhen or shiquan dabu decoction $(p<0.01)$. For group F, T-cell activity was significant different to group $G$, which suggested that the drug pair of astragali and cinnamon had effect of improving T-cell activity in shiquan dabu decoction. For B-cell activity, it had no this effect.

\section{Discussion}

It is well known that qi or/and blood deficiency can lead to decreased immune function (Wang et al., 2009). Therefore, the focus of the work is to explore the immunological mechanism of the drug pair in shiqu dabu decoction. Immune system is composed of immune organs, immune cells and immune molecules, in which immune cell is as executor of immune response.

WBC is closely related to immune. Neutrophil, as one kind of WBC, has strong phagocytosis belonging to non -specific immunity. In Human blood system, more than $95 \%$ of complement receptor one (CR1) is presented in $\mathrm{RBC}$. The probability of removing the antigen-antibodycomplement complexes by RBC is 500 600 times larger than it by WBC (Yang et al., 2009); therefore, RBC is also important for immune. In this work, the number of WBC and RBC in mice with qi deficiency and blood deficiency were lower than normal, which was consistent with the illness in clinic. When they were intervened by these two compound recipes, the number of WBC and RBC were all enhanced, which confirmed their pharmacological action recorded by ancient medicine book. In the status of qi deficiency, Shiquan dabu has ability in enhancing WBC, which suggested the drug pair of astragali and cinnamon has a synergistic effect with bazhen decoction. However, the drug pair had not ability in increasing the number of WBC.

$\mathrm{PP}$ is one kind of peripheral immune organs, it plays role in intestinal immunity. When antigen is engulfed and transferred into $\mathrm{PP}$, the process including of antigen presentation, recognition, immune cell activation and proliferation take place; finally the immune function is enhanced. In addition, immune tolerance can also be induced by food antigen. Thus, the functional status of PP is associated with immune function. The number of PP will decrease when immune function decline. In this study, the number of PP in Mice with qi or blood deficiency was lower than normal, which reflected that their intestinal immunity declined. When they were administrated with bazhen decoction, the PP was improved in status of blood deficiency; however, it was not improved in status of qi deficiency. The number of PP was all increased when siquan dabu was administrated, which suggested that the drug pair of astragali and cinnamon has a strong role in the improvement of PP, through which intestinal immunity was enhanced. 
$\mathrm{T}$ lymphocytes are composed of cytotoxic $\mathrm{T}$ lymphocytes (CTLs) and T helper (Th) lymphocytes. CTLs can kill virus-infected cells or tumor cells. Th lymphocytes have regulation effect in immune response. B lymphocytes can capture antigen and present to Th cells to induce immune response, it can also be differentiated to plasma cell to secret antibody to induce humoral immune response. Therefore, $\mathrm{T}$ and $\mathrm{B}$ Lymphocytes play vital role in the immune system. In this work, the B - and T-cell activity all declined in status of qi and blood deficiency, which suggested that decreased immunity was occurred. When bazhen and shiquan dabu decoction was administrated, the activity of T, B was improved. Especially for T-cell, it was highly improved, which suggested that the drug pair of astragali and cinnamon in shiqu dabu decoction has stronger activity in enhancing T-cell function. However, it has not this action for B-cell.

Peritoneal macrophages as a kind of antigen presenting cells can capture the antigen and process it into antigenic peptide, and MHC combine antigenic peptide to form complex, which is transferred on the cell membrane, which is recognized by TCR of T-cell to induce immune response. Therefore, the phagocytic activity of peritoneal macrophages is related to immunity function. In this work, bazhen and shiquan dabu decoction all had the action of the improvement of the phagocytic activity of peritoneal macrophages. However, the drug pair of astragali and cinnamon has not effect in shiquan dabu decoction.

The main chemical compositions of astragali were polysaccharides, saponins, flavonoids, amino acids and so on. Improvement of immune function, antioxidation, anti-radiation and anti-cancer have been reported. Cinnamon has a role in regulating immune function too. For instance, since the occurrence of asthma is related to immune dysfunction, in asthma patients, Th2 type cytokines are dominant and induce rather strong humoral immune response. Cinnamon can correct the imbalance of cytokines through downregulating Th2 type cytokines IL-2 and IL-5 levels.

Theses documents indicated that astragali and Cinnamon had many actions in regulating immunity of body. Results in this work also suggested that the drug pair has some actions in shiquan dabu decoction. However, the mechanisms of them have not been clarified and further studies should be carried out..

In conclusion, the drug pair of astragali and Cinnamon in shiquan dabu decoction had some pharmacological actions. Chinese medicine theory thinks that medicine used should be based on symptoms. The results of this work were consistent with this theory; the drug pair astragali and cinnamon in shiquan dabu decoction has different pharmacological action in regulating body function. Therefore, the quantity of drug pair in shiquan decoction should be changed according to the symptoms in process of diseases.

\section{Acknowledgements}

This study was supported by start-up foundation of Jiangsu University of Science and Technology (No. 2009sw150J and No.35211101).

\section{References}

Cao Y, Xia QH, Meng H, Zhong AP. Pharmacological effects of serum containing Chinese medicine Bushen Huayu Jiedu compound recipe in lung cancer drug-resistance cells. Chin J Integr Med. 2008; 14: 46-50. PMID: 18219453

Chen LY, Luo X. Effects of Zhigancao Decoction on arrhythmia with syndrome of deficiency of both Qi and blood in rats. Shizhen Guo Yi Guo Yao. 2010; 21: 1631-33.

Chen Y, Li XR, Zhao J, Zhou T, Zou Q. Chemical component analysis of volatile oil in drug pair Herba EphedraeRamulus Cinnamomi by GC-MS and CRM. J Cent South Univ Technol. 2007; 14: 509-13.

Li XD, Pan WS, Nie SF, Wu LJ. Studies on controlled release effervescent osmotic pump tablets from Traditional Chinese Medicine Compound Recipe. J Controlled Release. 2004; 96: 359-67. PMID:15120893

Liu Y, Li XH. Preliminary molecular basis of Danggui-buxuetang on Qi deficiency and blood stasis syndrome. J Shenyang Pharm Univ. 2008; Z1: 110-11.

Song HT, Guo T, Zhang RH, Zheng CL, Ma Y, Li X. Preparation of the traditional Chinese medicine compound recipe heart-protecting musk $\mathrm{pH}$-dependent gradientrelease pellets. Drug Dev Ind Pharm. 2002; 28: 1261-73. PMID: 12476872

Sun E. Changes in some elements, enzymes and energy charge in skeletal muscle of rats with spleen qi deficiency. Chin J Integr Med. 1993; 13: 736-38. PMID:8136649

Wang YJ, Shi Q, Jiang JC, Bian Q, Liang QQ, Li CG, Zhou Q, Cui XJ, Lu S, Zhou CJ. Mechanism of Yiqi Huayu Bushen recipe in treating cervical syndrome in rats with qi deficiency, blood stasis and kidney deficiency. Chin J Integr Med. 2009; 7: 53-58. PMID: 19134458

Yang LH, Qiu JD, Li HQ. Effects of astragalus heteropolysaccharides on erythrocyte immune adherence function of mice with adjuvant-induced arthritis. Acta Pharma Sin. 2009; 44: 1364-70. PMID: 21351470

Yang QL. Determination of sucrose in shiquan dabu gao by optical rotation. Zhong Yao Tong Bao. 1986; 11: 32-33. PMID: 2952352

You QY, Wang P. The studies of Ziziphi Spinosae Decoction (ZSD) intervention to old rats syndrome model with Yin and blood-deficiency (sic) and sleep deprivation. J Pharmacol Sci. 2009; 109: 247.

\footnotetext{
Author Info

i Hui Yan (Principal contact)

"e-mail: yanh1006@163.com
} 